\title{
Designing with Alganyl
}

\author{
Fiona Bell \\ fiona.bell@colorado.edu \\ ATLAS Institute \\ University of Colorado Boulder \\ Boulder, Colorado, USA \\ Ella McQuaid \\ ella.mcquaid@colorado.edu \\ Mechanical Engineering \\ University of Colorado Boulder \\ Boulder, Colorado, USA
}

\author{
Latifa Al-Naimi \\ lateefah.alnaimi@colorado.edu \\ Computer Science \\ University of Colorado Boulder \\ Boulder, Colorado, USA \\ Mirela Alistar \\ mirela.alistar@colorado.edu \\ ATLAS Institute and Computer Science \\ University of Colorado Boulder \\ Boulder, Colorado, USA
}
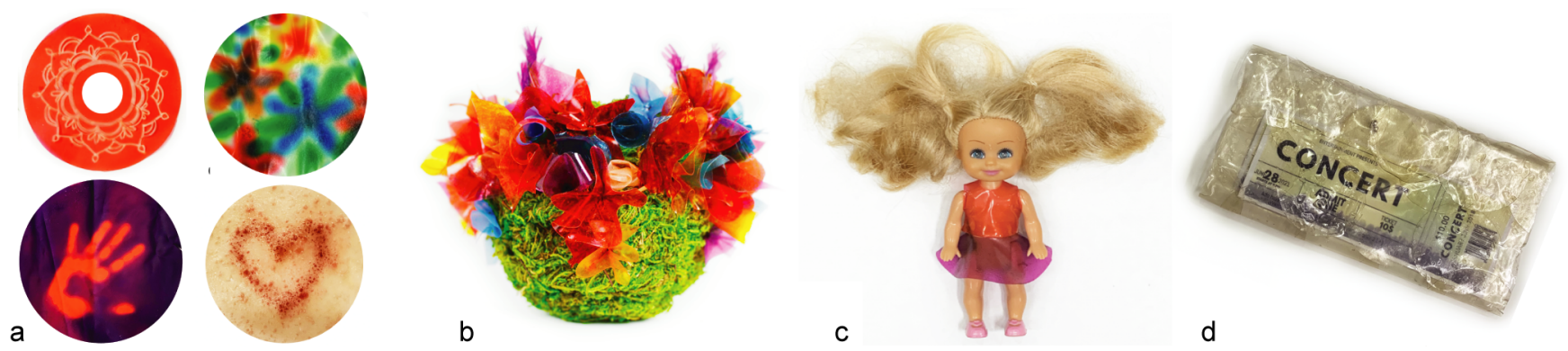

Figure 1: (a) We explored designing with Alganyl, a vinyl-like material made from marine algae, by embedding color, patterns, texture, thermosensing and photosensing. We used ALGanyL to made a wide range of applications including (b) a floral sculpture, (c) doll clothing, and (d) a transparent concert bag.

\begin{abstract}
We explore designing with ALgANYL, a highly customizable and sustainable biomaterial made of food-grade ingredients. To make ALGANYL, we optimized existing bioplastics for flexibility and strength, resulting in a biomaterial that acts and feels similar to vinyl fabric. Moreover, Alganyl is a zero-waste material that can be fully recycled or biodegraded in 60 days. We further explored designing with Alganyl by embedding color, texture, and sensing pigments. We showcase a wide range of applications made entirely of ALGANYL including a sculpture, doll clothing, environment-sensing accessories, and a recycled concert bag. We conducted a user study with 12 participants that made ALGANYL at home, fabricated their own applications, and then speculated on future applications for Alganyl. Overall, participants found Alganyl to be an accessible biomaterial that can be used for many applications. We hope designers and HCI researchers will embrace ALGANYL as a sustainable biomaterial for making recyclable and biodegradable artifacts.
\end{abstract}

This work is licensed under a Creative Commons Attribution International 4.0 License.

TEI '22, February 13-16, 2022, Daejeon, Republic of Korea

(c) 2022 Association for Computing Machinery.

ACM ISBN 978-1-4503-9147-4/22/02.

https://doi.org/10.1145/3490149.3501308

\section{CCS CONCEPTS}

- Human-Computer Interaction $\rightarrow$ Interface design prototyping.

\section{KEYWORDS}

biomaterials, bioplastic, biodesign, sustainability, user study

ACM Reference Format:

Fiona Bell, Latifa Al-Naimi, Ella McQuaid, and Mirela Alistar. 2022. Designing with Alganyl. In Sixteenth International Conference on Tangible, Embedded, and Embodied Interaction (TEI '22), February 13-16, 2022, Daejeon, Republic of Korea. ACM, New York, NY, USA, 14 pages. https://doi.org/10. $1145 / 3490149.3501308$

\section{INTRODUCTION}

Due to the rising concerns regarding the environmental impact of plastics in the packaging, fashion, and electronics industries, designers, material scientists, and HCI researchers have started to explore new materials and methods that consider designing with sustainable alternatives to plastic. Popular biodegradable plastic alternatives include mycelium [17, 31, 99], kombucha SCOBY (symbiotic culture of bacteria and yeast) $[8,61,71]$, and a variety of biocomposites that have plastic or leather-like qualities [57, 68, 81, 96]. While mycelium and kombucha SCOBY have already been explored in the context of HCI, this work contributes a bioplastic called ALGANYL, a highly customizable material made up predominantly of an algae-based powder called agar. Like most biomaterials ALGANYL 
can be disposed of in the environment-biodegradability tests show that Alganyl takes about 60 days to fully degrade in soil-however, Alganyl distinguishes itself from other biomaterials by being a circular material that is easily recycled via a zero-waste re-cooking method. Thus, designers can choose to give used ALGANYL a second life, or dispose of AlgANYL via biodegradation, both options having minimal impact on the environment.

Alganyl is based on Margaret Dunne's agar bioplastic recipe [30], containing a mixture of agar powder, water, and glycerin [30]. We expanded upon Dunne's work by first optimizing the ratio of ingredients for our desired strength and flexibility, which results in a material that is most similar to vinyl fabric; strong, flexible, slightly stretchy, and feels smooth against the skin. We then utilized our optimized ALGANYL recipe to explore a set of design parameters that allow the user to customize their Alganyl via color, texture, textile fabrication techniques, sensing ability, and electrical conductivity. Furthermore, we applied our five-step design process (cooking, drying, fabricating, re-cooking, and disposing of) to create a wide range of applications including a floral sculpture (Figure 1b), doll clothing (Figure 1c), environment sensing accessories (a UV light sensing bracelet and temperature sensing necklace), and a transparent concert bag made from re-cooked bioplastic (Figure 1d).

Next, we wanted to study how people who are unfamiliar with making bioplastics would perceive it. Would theytreat it as a textile, as a utilitarian plastic, or another material? What kind of applications would they make with it? We also wondered how easily Alganyl could be made and applied to unique applications by unfamiliar users to determine the accessibility of AlganyL. We also wanted to gain a deeper understanding of how users felt about designing with Alganyl so that we could more easily examine the features and drawbacks of the material. To answer these questions, we held a workshop for 12 university students, where we taught them about Alganyl and how to make it. The workshop attendees were then asked to make any application of their choosing from the AlgANYL they made during the workshop and fill out a post-workshop survey. In this study, we specifically use ALGANYL as a technology probe [42] to (1) field test ALGANYL as a new biodegradable material, (2) gain insight into the needs and desires of the users, and (3) inspire users to think of new applications for Alganyl. Thus, we focus primarily on the made and envisioned applications for Alganyl, the accessibility of making AlganyL, features of ALgANYL the users liked, and barriers users had with Alganyl. Ultimately, our findings indicated that most participants appreciated the simplicity of the making process and the biodegradability of the material. Moreover, participants were easily able to envision numerous applications for ALGANYL ranging from the highly artistic to the purely utilitarian.

In this paper, we provide a set of techniques that can be used by HCI researchers to design with Alganyl. We present in detail how we explored the design space by embedding various colors, textures, sensing, and electronics in Alganyl through subtractive (i.e., cutting) and additive (i.e., thermoadhesion) manufacturing methods combined with traditional textile techniques. We demonstrate how these techniques can be used to make biodegradable artefacts including a floral sculpture (Figure 1a), clothing for dolls (Figure 1b), environment-sensing accessories (a UV light sensing bracelet and a heat-sensing necklace), basic biodegradable circuits, and a bag made from re-cooked Alganyl (Figure 1c). To gain insight on Alganyl's accessibility and its potential future applications, we conducted a user study with 12 participants that made ALGANYL at home, then designed and created their artefacts. Lastly, we discuss the limitations of AlgANYL, we highlight the importance of experimentation and experience prototyping [18] during designing with AlganYL, and we speculate on the future implications of AlgANYL. Through this work, we ultimately hope to encourage designers and HCI researchers to embrace ALGANYL as a sustainable biomaterial that can be used to create a wide variety of reusable and biodegradable applications.

\section{RELATED WORK}

Recently, HCI researchers have begun incorporating biological materials into their practices, resulting in a range of interfaces that include displays [13, 53, 65, 92], art [4, 54, 75], instruments [51], environments [70], systems [11, 40, 60], games [22, 49, 50, 73], robots [20, 77], and wearable devices [7, 32, 71, 99, 101, 102]. Moreover, the "material-turn" in HCI [84] encourages researchers to take inspiration from materials themselves to arrive at a design concept, as opposed to forcing a material to fit a predetermined design concept. Notably, Karana et al. introduced the Material Driven Design methodology [48], Pedgley et al. proposed working with materials by focusing on designing material experiences [78], and Parisi et al. further addressed how to gain material understanding with their approach of Material Tinkering, which highlights the importance of hands-on experimentation and iteration [76]. Our work contributes to this growing field of biology and material-centered HCI with a series of methods, design parameters, and applications for a sustainable biomaterial, AlgAnyL.

\subsection{Bioplastics}

To meet society's plastic needs, the world has been producing around 300 million tonnes of plastic every year since 2015, which is nearly equivalent to the weight of the entire human population [33]. These petroleum-based plastics take about 500 years to decompose in a landfill while releasing toxins into the environment [83] such as bisphenol A (BPA) and polystyrene-based (PS) oligomers, both of which are linked to disrupting animal hormones and damaging their reproductive systems [14]. Motivated by these environmental issues, biodegradable plastics (or bioplastics) aim to provide a more sustainable alternative to petroleum-based plastics. Bioplastics can be made from a range of highly abundant starches like potatoes [97], corn [29, 33], wheat gluten [28], cassava [95], woodflour [69], and algae [39, 66, 91, 93]. We specifically introduce an optimized version of Margaret Dunne's recipe for an agar (algae powder) bioplastic [30] that we call ALgANYL.

\subsection{DIYBio}

The Do-It-Yourself (DIY) movement encourages people to make items themselves rather than purchasing items or paying someone else to make the item [56]. This practice encourages independent thinking and problem solving [89]. In recent years, the DIY movement has even merged with biological sciences (i.e., DIYBio), which 
are notoriously inaccessible because that they typically require specialized equipment, training, and knowledge. However, advancements in biological sciences paired with the efforts of the DIYBio community to make biological tools and procedures accessible have lead to increased DIYBio experimentation [27, 55]. Moreover, the resources for the DIYBio community have flourished-from community wet-labs that provide equipment [59] to online instruction manuals [44]. The rise of DIYBio has also prompted collaborations with the human-computer interaction (HCI) community to create a wide range of interactive interfaces $[4,11,40,71,73,99,102]$.

\subsection{DIY Biomaterials}

DIY biomaterials have gained significant traction in the HCI community over the past few years. Most notably, Lazaro Vasquez used a commercial DIY mycelium kit [63] to create interactive accessories [99] and breadboards [98], while $\mathrm{Ng}$ used DIY kombucha broth recipes $[21,94]$ to create SCOBY wearables and origami [71]. Similarly, our recipes and methods were primarily inspired by opensource work such as Dunne's Bioplastic Cook Book [30], Kwong's Bio-Plastic Handbook [57], Pepin's Research Book Bioplastics [80], The Chemarts Cookbook [58], Kretzer's "Gelatin-based Bioplastic" tutorial [52], Raspanti's "BioFabricating Materials" tutorial [81], and Materiom's "Material Library" [68]. More specifically, we optimized Dunne's agar bioplastic recipe to create AlgANYL, a biomaterial that aligns itself with works such as AlgiKnit's seaweed-based bioplastic yarn [3], GreenGear's sugarcane-based bioplastic "EcoRain Poncho" [12], and McCurdy's algae-based bioplastic "Carbon-Negative" raincoat [26]. AlgANYL differentiates itself from these works by incorporating thermochromic and photochromic pigments, which are commonly used in the e-textiles community to create a wide range of interactive wearables [15, 25, 41, 45, 47, 74, 79]. These pigments make AlgANyL more interactive without impacting its ability to biodegrade $[10,19]$. We also used activated charcoal, a biodegradable conductive material $[34,88]$ to create our circuits instead of wires like other interactive biomaterials explored in HCI [71, 99].

\subsection{Sustainability}

Sustainability in Human-Computer Interaction (SHCI) has been addressed through Sustainable Interaction Design (SID), which links invention to disposal and promotes renewal and reuse [16], and Sustainability in Design, which considers energy, waste reduction, and product reuse on an individual, group, and societal level [67]. Moreover, researchers have discussed ways of evaluating sustainability throughout the design process by examining limitations of current practices [82] and by applying the Life Cycle Analysis-a cradleto-grave method to evaluate potential environmental impact-to sustainable prototyping [100]. In this work, we primarily focus on the end-of-life of ALGANYL by developing a recycling technique that prolongs the life of the material, and by testing AlganyL's biodegradability.

\section{MATERIAL CHARACTERIZATION}

\subsection{Process}

Our design process for Alganyl consists of five steps illustrated in Figure 2: cooking, drying, fabricating, re-cooking, and disposal.
Cooking consists of bringing the mixture of agar, water, and glycerin (see amounts of ingredients in Table 1) to a boil by either microwave, which takes 2 minutes on high, or stove-top, which takes 10 minutes on high. During this cooking process, the mixture should be stirred every 15 seconds if using a microwave or constantly if using a stove top. Once transformed into a uniform, viscous mixture, we poured the heated Alganyl onto a clean, flat, heat-resistive surface and left it to dry for 24-72 hours (dry time depending on airflow and humidity of the surrounding environment). During this drying time, the Alganyl shrinks approximately $46 \%$ in volume due to the evaporation of the water. We then used cutting and heat sealing as our primary fabrication techniques to create applications from dry Alganyl. We used X-Acto knives, scissors, and laser cutters to manufacture Alganyl subtractively. To manufacture Alganyl additively, we used fabric irons, plastic sealers, and the hot tip of glue guns. When AlganyL is used and no longer wanted, we account for two disposal methods. The first, a circular disposal method, involves re-cooking dry ALgANYL scraps in additional water that re-starts the process at the drying stage. The final disposal method involves biodegrading AlganyL in the natural environment.

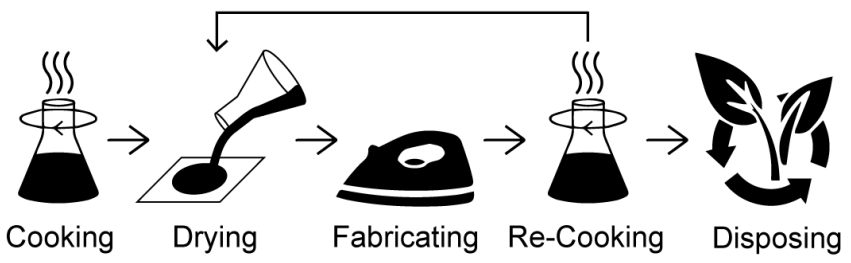

Figure 2: Our sustainable design process for Alganyl consists of five steps: cooking, drying, fabricating, re-cooking, and disposing.

\subsection{Flexibility and Strength}

As mentioned, Alganyl is made by mixing and cooking a mixture of water, agar, and glycerin. We began our investigation by optimizing our recipe by testing various ingredient ratios that we showcase in Table 1. For each ratio, we ran tensile tests according to the ASTM D638 Type IV standard for tensile testing plastics [6] to determine the flexibility and strength of each sample-the values in columns 4 and 5 being the average of five tested samples. For these tests, we laser cut the Alganyl into the prescribed dog bone shape and then placed it in a universal testing machine set up with a $5 \mathrm{kN}$ load cell, which stretched the sample at a rate of $0.1 \mathrm{~mm} / \mathrm{min}$. In column 4 , we present the average values for the percent elongation at break (i.e., flexibility). In column 5, the average values for tensile strength were calculated using the formulas dictated by the ASTM D638 Type IV standard [6]. As expected, the results show that the concentration of glycerin, a commonly known plasticizer [85], is the main factor in the flexibility and strength of ALGANYL. Specifically, these properties have an inverse relationship-an increase in glycerin leads to increased flexibility but a decrease in strength. We decided to use the recipe in Table 1, row 3 for the remainder of the work because it provided the best balance of strength and flexibility. Moreover, the ratio in row 3 provided the best feeling for 
the material, its flexibility allowing the smooth material to mold well to the skin without feeling too sticky.

\begin{tabular}{ccccc}
\hline Water $(\mathrm{mL})$ & Agar $(\mathrm{g})$ & Glycerin $(\mathrm{g})$ & Elongation (\%) & Strength $(\mathrm{MPa})$ \\
\hline 50 & 2 & 0 & 0.61 & 56.67 \\
50 & 2 & 1.5 & 6.09 & 31.66 \\
$\mathbf{5 0}$ & $\mathbf{2}$ & $\mathbf{3}$ & $\mathbf{1 3 . 0 4}$ & $\mathbf{1 2 . 5 3}$ \\
50 & 2 & 4.5 & 16.87 & 6.45 \\
50 & 2 & 6 & 19.65 & 4.19
\end{tabular}

Table 1: We calculated the flexibility, denoted in the table as Elongation \%, and strength of five different ingredient ratios. We chose the ratio described in row 3 as our primary $A_{L}$ GANYL biomaterial because it provided a balance of strength and flexibility.

\subsection{Sensing Alganyl}

Having the Alganyl recipe determined, we further characterize its sensory aspects; specifically, AlganYL's look, feel, smell, and taste.

Look and Feel. Alganyl looks most similar to the transparent, flexible, petroleum-based plastics used for Ziploc bags, which caused many participants from our user study to see ALGANYL as an alternative for single-use plastic bags. However, AlganyL is slightly thicker, making it feel more sturdy and durable. Therefore, the closest traditional material we could find to ALGANYL in terms of look and feel was vinyl fabric [46], which we compare in Figure 3. Similar to vinyl, Alganyl has a shiny sheen to its surface and feels very smooth. The look and feel of ALgANYL can also be easily customized, which we explore in Section 4.
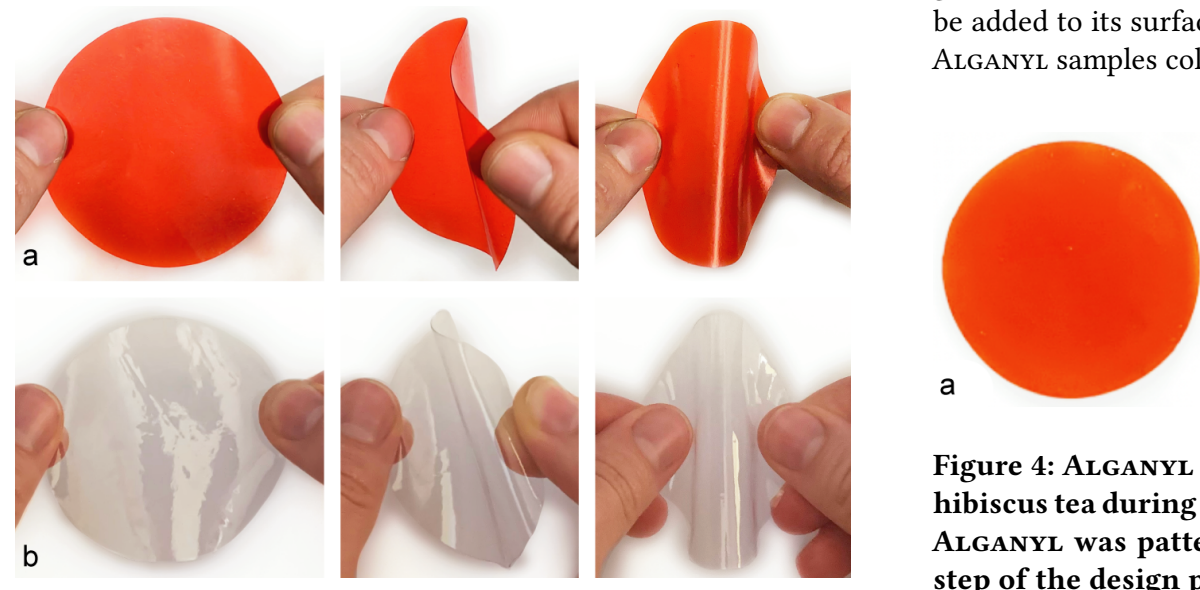

Figure 3: (a) Alganyl in comparison to (b) vinyl.

Smell. Although we did not perform the necessary experiments to claim that ALgANYL's odor is not distinguishable by human olfaction, we observed that we could not distinguish any specific odor when working with AlgANYL. We also did not get any odor reported by the study participants.

Taste. Similarly, we do not have enough scientific support to claim that Alganyl is safe to eat. However, the basic Alganyl
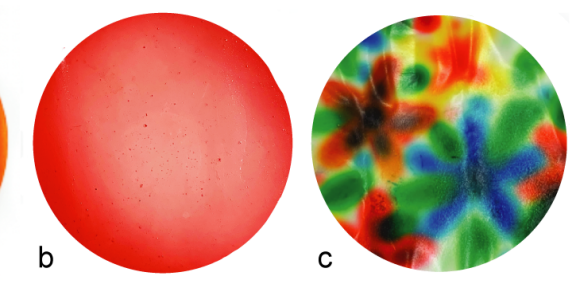

Figure 4: Alganyl was colored with (a) red food dye and (b) hibiscus tea during the cooking step of the design process. (c) Alganyl was patterned with food color during the drying step of the design process.

samples are made of food-grade agar, and glycerin, and similar agar-based bioplastics have been reported to be edible [2, 23, 62]. For the electronic circuit described in Section 4.5, we used foodgrade activated charcoal. There have been no studies regarding the edibility of the photochromatic and thermochromatic pigments we used for adding sensing capabilities, as we describe in Section 4.4. In terms of their toxicity and biocompatibility, similar pigments have been proposed for biomedical applications [19].

\subsection{Color}

We experimented with dying Alganyl with synthetic and natural pigments to obtain either uniform or patterned coloring, as presented in the following.

Mono Color. Colored Alganyl, as seen in Figure 4a, can be obtained by adding 2-3 drops of synthetic food-grade dyes during the cooking process. On the other hand, natural dyes are commonly extracted using a solvent (i.e., the dye itself is a solution that may have a very low pigment concentration). In that case, the natural dye solution can be used to substitute the water in the ALGANYL recipe. Figure $4 \mathrm{~b}$ depicts an Alganyl sample that substituted water for hibiscus tea during the cooking process. While the red food dye produced a uniformly colored sample, the hibiscus tea produced a red sample that was darker in color around the edges and lighter in the center (Figure 4b).

Patterned Color. We also experimented with creating multicolored patterns by painting food dye onto the surface of AlgANYL during the drying step. The dyes are most effectively applied to ALGANYL when the mixture has jellified but has not thoroughly dried, which happens approximately 30 minutes after cooking. When the gel is stable (i.e., it does not move when touched), food dye can be added to its surface with fingers or brushes. Figure $4 \mathrm{c}$ depicts AlgANYL samples colored in a tie-dye flower pattern.

The addition of the dyes we used for both our uniform and patterned samples did not change the original flexibility and strength of the Alganyl. Moreover, our Alganyl samples kept their color and patterns for over two years at indoor temperature $\left(\max 25^{\circ} \mathrm{C}\right)$ under artificial lighting conditions. When left in direct sunlight for more than a few days, we noticed that the ALGANYL's color fades similarly to clothing dyed with organic dyes. Re-cooking monochromatic AlgAnyl samples did not affect the initial color

\section{DESIGN SPACE}


intensity. However, as expected, re-cooking patterned or multicolored AlgANyL resulted in a uniform color obtained by mixing all pigments.

Example Application. We used a variety of colored AlgANYL samples to create a floral sculpture, seen in Figure 5. To make the sculpture, we rolled, folded, twisted, and fused colored samples of Alganyl to shape them into tridimensional flowers. We then anchored each flower with needle and thread to the wire scaffolding of a living moss nest. Our half living, half man-made sculpture questions the "natural" aspects of our work. The Alganyl flowers biodegrade, similar to living (organic) flowers, and follow the perennial rhythm of plants, but do not have an inherent life source and are wholly artificial.

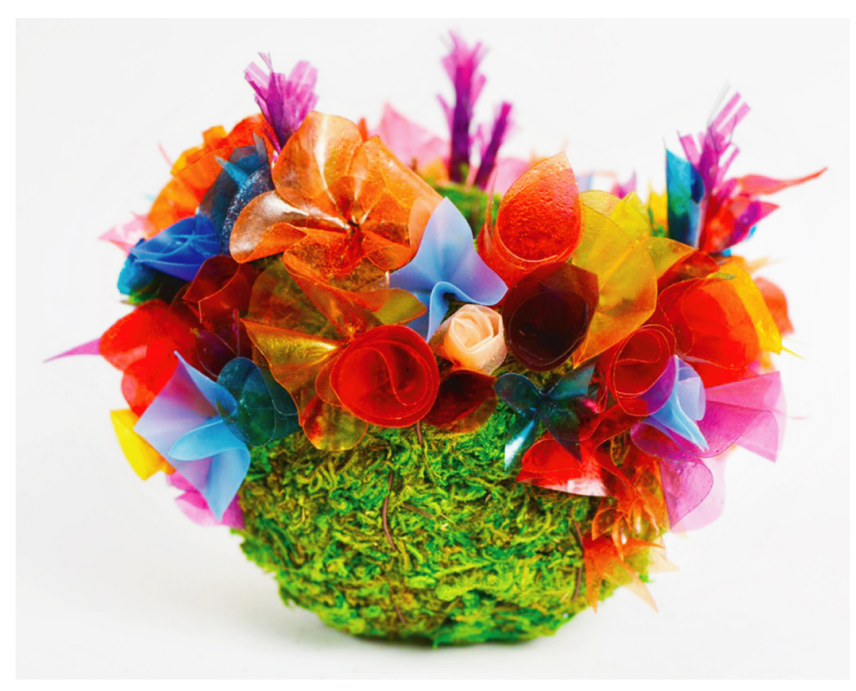

Figure 5: Our Alganyl sculpture is made up of a variety of colored samples that were transformed into "flowers" that we anchored to a living ball of moss.

\subsection{Texture}

We experimented with applying texture to ALGANYL through molding during the drying process and through laser engraving (subtractive manufacturing).

Drying. We dried the liquid Alganyl on a textured surface rather than a flat surface, such as a Blokus game board [1] (Figure $4.2 \mathrm{a}$ ) and bubble wrap (Figure $4.2 \mathrm{~b}$ ). Once poured onto the textured surface, the Alganyl takes the same amount of time to dry as it would on a flat surface. We also noticed that the height of the drying surface affected the resulting AlganyL. For example, the height of the air bubbles of the bubble wrap exceeded the natural thickness of the drying Alganyl, leading to a circular fishnet texture. Meanwhile, the Blokus board did not exceed the natural height of ALGANYL leading to a sample with a checkered texture. As shown in Figure 6, the bubble wrap sample had the same texture on both sides of the AlganyL, while the Blokus board sample had one smooth surface on the side that was exposed to air and one textured side that was touching the drying surface.
Laser Engraving. After ALGANYL dried, we engraved the desired pattern into its surface, as shown in Figure 6c. We used the following laser settings: $70 \%$ speed, $20 \%$ power, and $50 \%$ frequency. Similar to the Blokus board sample, the engraved sample had one smooth side and one side that was textured.
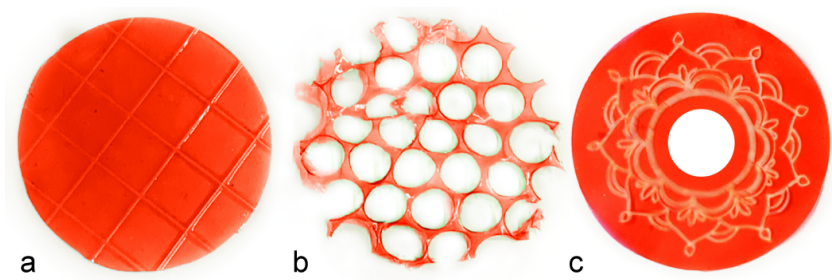

Figure 6: We textured Alganyl by drying it on textured surfaces like (a) a Blokus game board [1] and (b) bubble wrap or by (c) engraving dry Alganyl with a laser cutter.

While adding texture most clearly changed the feel of the ALGANYL, in some cases, the texture also impacted the strength and flexibility. For example, the sample in Figure 6a maintained the same strength and flexibility as the original ALganyl. In contrast, the sample in Figure $6 \mathrm{~b}$ lost a significant amount of strength but gained significant flexibility. The laser-cut sample in Figure $6 \mathrm{c}$ falls somewhere between these two samples, only slightly losing strength and gaining flexibility due to the etching and the hole in the middle.

\subsection{Textile Techniques}

Next, we explored how ALgANYL can accommodate traditional, fiber-textile fabrication techniques by creating 4 different swatches that employ weaving, knitting, pleating, and braiding, respectively.

Weaving. As shown in Figure 7a, we wove strips of AlgANYL to create a colorfully patterned swatch. While traditional weaving ties the threads together to keep the weave from falling apart, we used heat sealing to combine the woven pieces of Alganyl. While the final woven swatch acts like one congruent piece, the surface feels textured and more durable. Moreover, the woven piece is similar in flexibility but less stretchable than the original ALGANYL.

Knitting. As shown in Figure 7b, we knit Alganyl "yarn" into a swatch. To create the yarn, we cut Alganyl into thin strips (approximately $0.2-0.5 \mathrm{~cm}$ in width), which were plyed (i.e., twisted into a rope-like structure with a $0.1 \mathrm{~cm}$ diameter) and then heat sealed to hold their shape. We then used a garter stitch to knit our Alganyl yarn into a three-dimensional swatch. While the nonuniformity and occasional stickiness of the ALGANYL yarn made knitting difficult at times, the final swatch had an interesting texture and could be significantly stretched with little force. While easily stretchable, the knit structure felt delicate rather than durable-it felt like the yarn would rip if too much force were applied when stretching. As for the flexibility, the dimensionality of the knit structure impeded the movement of the swatch, making it harder to fold, twist, and crumple like the original AlganyL.

Pleating. As shown in Figure 7c, we pleated Alganyl to create a swatch with dimensionality. Pleating is a standard method in garment making to add volume to two-dimensional textiles. We specifically implemented the box pleat, which utilizes origami-like 
folds that were heat-sealed at the top of the AlganyL swatch to keep the pleats in place. While not having a texture like the woven or knit swatches, the pleated swatch is very tactile, being able to unfold when stretched and then automatically fold back in on itself when let go. As for strength and flexibility, the pleated AlganyL acted the same as the original AlganyL, except for the heat-sealed portions, which were much stronger and less flexible.

Braiding. As shown in Figure 7d, we braided eight strands of Alganyl to create an intricate and robust sample of flat rope (also known as webbing). While braiding is typically associated with hair or bread making, we employed braiding as a technique to selectively pattern different colors of ALgANYL, and to add texture and significant strength. Due to the robustness of the braided AlganyL, the flexibility was impacted; specifically, the braided AlgANyL resisted actions like folding, where the sheer amount of material got in its own way.
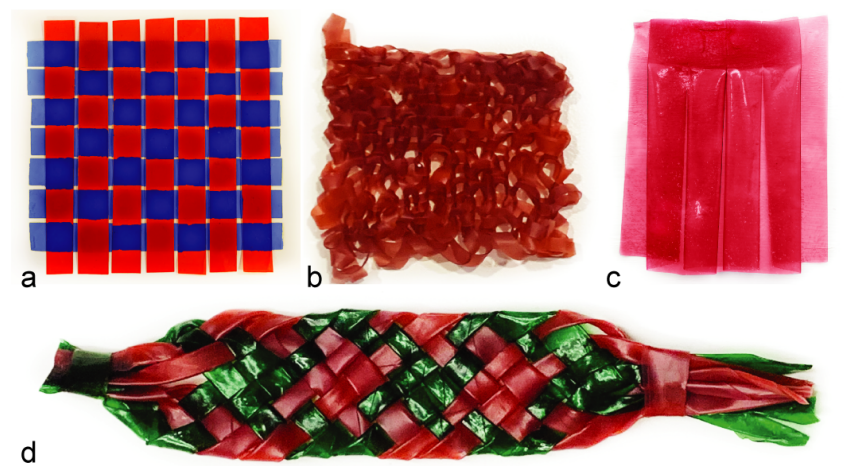

Figure 7: We explored the traditional textile fabrication methods of (a) weaving, (b) knitting, (c) pleating, and (d) braiding to add texture, pattern, strength, and dimension to Alganyl.

Example Application. While Alganyl can be easily customized via the color and shape of the clothing, we see our textile techniques providing another creative opportunity for clothing. Customizability plays a vital role because it allows designers to use their imagination when making clothing. We created a number of pieces of clothing for dolls and action figures (Figure 8). We specifically used a simplified braiding technique to create a belt (Figure 8a) and the pleating technique to create a doll skirt (Figure 8b). We also created several other colorful clothing items to dress different dolls fully (Figure 8c and d).

\subsection{Embedded Sensing}

Similar to our experiments with color, we experimented with embedding sensing properties in ALgANYL. We specifically looked at how to sense ultraviolet (UV) light and temperature using photochromic and thermochromic pigments. These sensing pigments change color when activated. They come in the form of powders, so we added them to the ALGANYL recipe during the cooking step. After some trial and error, we achieved the best results by adding 0.25 grams of powder to the ratios in Table 1 .

Photosensing. We used a photochromic powder that changes from light cream (not exposed to UV light) to dark pink (exposed
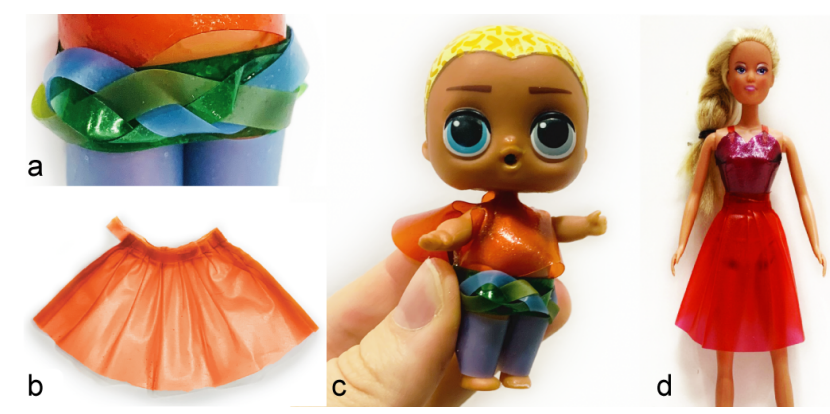

Figure 8: We made a variety of doll clothing including (a) a braided belt and (b) a pleated skirt for (c,d) two different kinds of dolls.

to UV light) purchased from Chromatic Technologies Inc [43]. We tested the sensing abilities of our photochromic AlganyL by placing it indoors (Figure 9a), indoors with a UV pen (Figure 9b), and outdoors in the sunlight (Figure 9c). While placing the photosensing ALgANYL outdoors turned the sample uniformly dark pink because of the UV rays in sunlight, the UV pen gave the user the ability to write and draw their own shapes and patterns. Once the sample was removed from UV light (i.e., the pen was turned off and the sample was inside), the photosensing Alganyl quickly returned to its cream color.

Thermosensing. We used a thermochromic powder that changes from white (hot) to dark blue (cold) purchased from Chromatic Technologies Inc [43]. We mixed this pigment with red food dye to create an AlganyL sample that is purple when at room temperature (Figure 9d). When the thermochromic sample is heated via body heat (Figure 9e), the sample changes to a red color (Figure 9f), indicating that it sensed the temperature change.
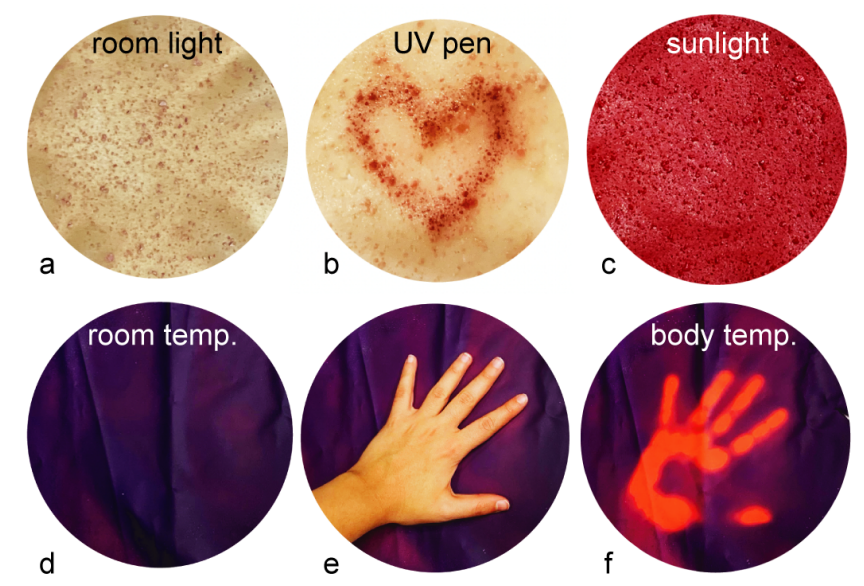

Figure 9: (a) Photosensing Alganyl is cream-colored when indoors, exposed to ambient light, and turns dark pink when (b) drawn on with a UV light pen, or (c) exposed fully to sunlight. (d) Thermosensing Alganyl is dark purple at room temperature, (e) but when heat is applied, (f) turns bright red. 
The addition of the pigments did not change the flexibility and strength of the Alganyl. As for the texture, the photochromic pigment was a very fine powder that did not affect the feel of the Alganyl. However, the larger grain size of the photochromic pigment gave the AlgaNYL a slight sandy feel. As mentioned above, the photochromic and thermochromic pigments we used are non-toxic and biodegradable, having been proposed for on-skin tattoos that indicate exposure to UV solar radiation [19]. We noticed that when re-cooked, photosensing and thermosensing samples of ALGANYL lose their sensing abilities. We assume this occurs due to exposure to higher temperatures for a long duration (re-cooking involves keeping the ALGANYL samples at boiling temperature until the material is dissolved in a homogenous mixture - usually takes more than 10 minutes).

Example Applications. We leveraged the UV light sensing ALGANYL to create a bracelet that acts as a sunblock reminder-sunlight having levels of UV light that damage skin. When the user is not exposed to UV rays (i.e., indoors), the bracelet is cream (Figure 10a), but when the user steps into the sun, the bracelet changes to dark pink (Figure 10b); the saturation of pink color depending on the amount of UV exposure. Because UV light is invisible, this bracelet helps visualize the amount of UV light the wearer is exposed to, regardless of the season. It is commonly assumed that sunblock only needs to be applied during the summer when it is hot. However, harmful UV light can be present regardless of the temperature (our bracelet seen in Figure 10b was worn during a cold winter day).

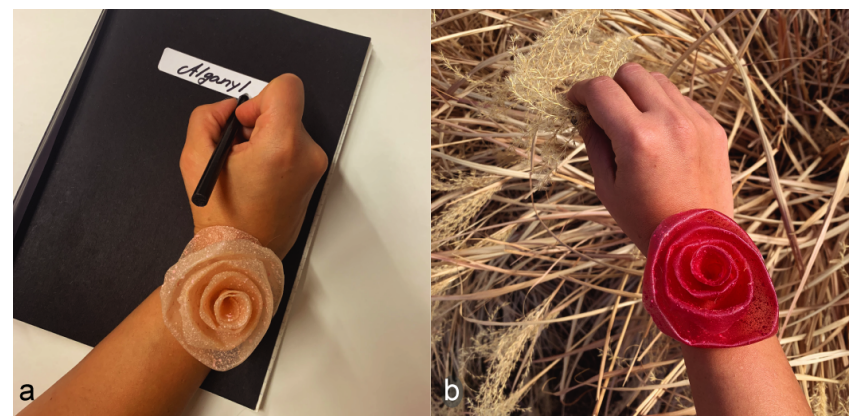

Figure 10: We applied our photochromic alganyl to a UV light sensing bracelet. (a) The bracelet is light pink when not exposed to UV light (i.e. when worn indoors) and (b) dark pink when exposed to UV light (i.e. when worn outdoors). The picture was taken during a cold winter day.

We applied our thermochromic Alganyl to a necklace that detects the temperature of the environment. When worn inside, the body's warmth and the room temperature turn the necklace light blue and orange (Figure 11a). When worn outside in the sun during the winter, the parts of the necklace close to the skin stays warm while the other parts get cold, leading to a range of color saturation (Figure 11b). When worn outside in the shade during the winter, the cold turns the necklace dark blue and orange (Figure 11a). While the necklace does indicate environmental conditions, its placement on the user's chest means that it more accurately indicates the temperature of the user wearing the necklace, thus showing the potential for creating biodegradable thermometers.

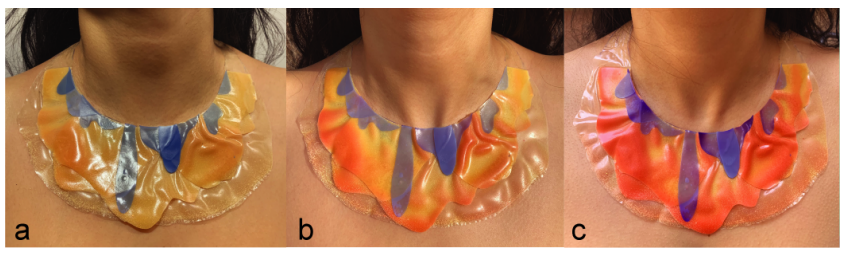

Figure 11: Thermochromic necklace worn (a) inside, (b) outside in the sun during the winter, and (c) outside in the shade during the winter.

\subsection{Electrical Conductivity}

Lastly, to create basic circuits, we explored adding an activated charcoal paste, a biodegradable carbon-based conductive powder mixed with vinegar $[34,88]$. We used a screen printing technique with a custom laser-cut stencil to "print" the charcoal paste onto the surface of the ALgANYL. Once printed, we heat-sealed another layer of ALgANYL on top to insulate and protect the charcoal paste from smudging, creating a charcoal paste sandwich.

LEDs. In Figure 12a, we embedded Light Emitting Diodes (LEDs) throughout our charcoal paste skyline and connected the sample to a power source. While the LEDs and external electronics are not biodegradable, we believe that these components can be easily removed after use and reused while everything else can be sustainably disposed of.

Thermosensing. We then combined our bare conductive circuit with our thermochromic AlgANyL (Figure 9) to create the sample seen in Figure 12b. For this sample, we utilized the same charcoal paste skyline circuit (minus the LEDs) and then heat-sealed thermochromic AlgANyl on top. When connected to a power source, the charcoal paste begins to heat up and selectively changes the color to reveal the city skyline.

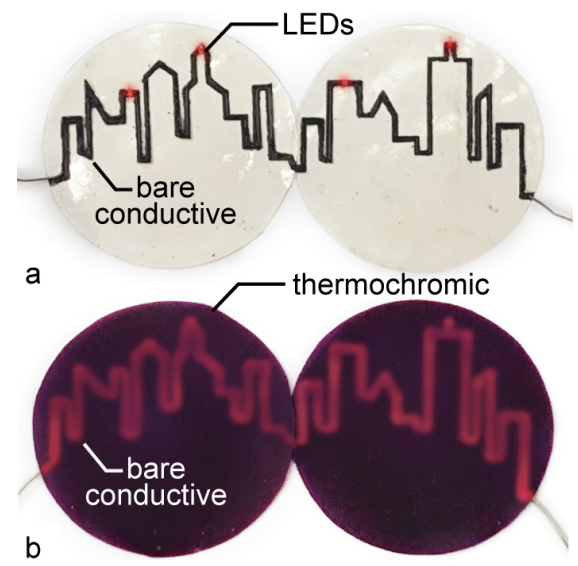

Figure 12: We used bare conductive to create biodegradable circuits that can (a) power a series of surface-mounted LEDs and (b) change the color of our thermosensing AlganYL. 


\section{DISPOSING AND DEGRADING ALGANYL}

\subsection{Recyclability}

We first explored a circular approach to disposal-recycling via our re-cooking method introduced in Section 3.1. For this method, we used a weight ratio of 3:10, ALgANyl to water. This mixture was then brought to boil and mixed until all the AlganyL dissolved (approximately 15 minutes on high), leaving a uniform mixture that was then poured, dried, and fabricated again. By implementing this re-cooking process, we can envision a circular economy using Alganyl, where the base materials (agar and glycerin) are re-used, eliminating the constant need for new base material.

The re-cooked ALGANYL measured a mass loss of $10 \%$, i.e., when we re-cooked 20g of ALGANYL scraps, we obtained 18.1g material. We noticed that when re-cooked, monochromatic samples of ALGANYL do not lose their color intensity, while multicolor samples of AlganyL result in a uniform mixed color. However, photosensing and thermosensing samples of Alganyl lose their sensing abilities when re-cooked. We assume this occurs due to exposure to higher temperatures for a long duration (re-cooking involves keeping the AlgAnyl samples at boiling temperature until the material is dissolved in a homogenous mixture-usually takes more than 10 minutes). As expected, when re-cooked, activated charcoal gets mixed with the rest of the material, so the resulting ALGANyL does not keep its original electrically conductive properties.

Example Application. We exemplify how re-cooked ALGANYL can find a second life with our clear bag for attending concerts, sports games, and other live events that commonly require a transparent bag to hold valuables for safety reasons. Such a requirement often adds the pressure of quickly finding ways to get a transparent bag for the sole purpose of using it during a concert. As seen in Figure 13, the transparent concert bag we made from re-cooked Alganyl was cut into sequins and heat-sealed into a composite piece to add pattern, texture, and durability. We then folded and heat-sealed the composite piece into the shape of the bag. Two additional sequins were added onto the bag to seal in two small magnets that kept the bag closed. While we used boiling water to re-cook ALGANYL for our bag, we found that non-boiling liquids that would come in contact with the bag for a short amount of time during the concert (i.e., spilled drinks and sweat) will not impact the structural integrity of the bag.
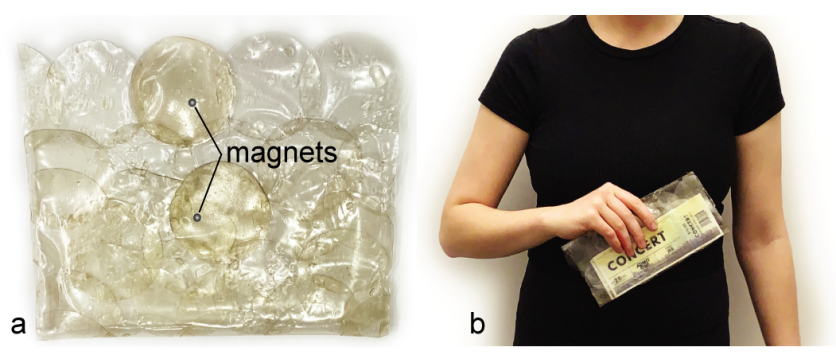

Figure 13: (a) Our concert bag is made up of re-cooked ALGANYL sequins heat sealed together, folded into shape, and embedded with small magnets that (b) holds essential items such as tickets, keys, phone, and money, and is carried as a clutch.

\subsection{Biodegradability}

Based on mass-loss over time measurements-a standard method to test the biodegradability of bioplastics [33]-we found that ALGANYL degraded $97 \%$ in 60 days in a controlled environment kept at $40^{\circ} \mathrm{C}$. Figure 14 shows ALGANYL's degradation qualitatively over 60 days. We note that ALGANYL might degrade at a different rate if disposed of directly in nature, depending on the soil's temperature, humidity, and microbial activity. The food-grade dyes and activated charcoal paste do not affect the biodegradability of the Alganyl, and the thermochromic and photochromic pigments we used have been shown to be consumed over time by macrophage cells having been used in biomedical applications [19].

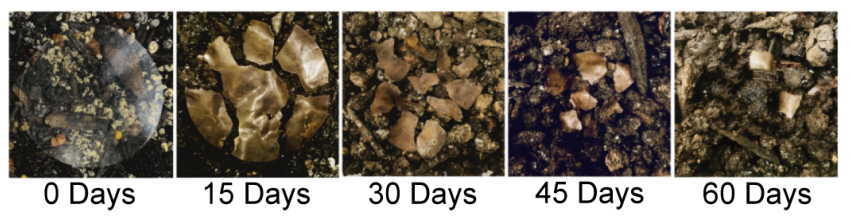

Figure 14: Alganyl biodegrades $97 \%$ in 60 days when buried in soil.

\subsection{Degradation in Different Environments}

When used indoors, under artificial light and room temperature, the Alganyl samples are robust: over two years, we did not notice any change in their properties (texture, strength, flexibility, color, and sensing). However, when brought outside, Alganyl faces some limitations, like the colored AlgANyL fading when placed in sunlight for long periods of time. However, the most significant limitation is water. ALGANYL absorbs water over prolonged periods (i.e., more than 20 hours), and it only dissolves in hot water (i.e., over $80^{\circ} \mathrm{C}$ ). When left in the rain for a prolonged time, AlganyL will only slightly absorb the water but will deform when drying (i.e., curl along its edges). In hot water, ALGANYL will fully dissolve, allowing it to be fully recyclable, as exemplified by our concert bag. This property was also taken advantage of by a participant in our used study who made ALGANYL detergent pods for the dishwasher (see Figure 15b).

\section{USER STUDY}

In this study, we used ALgANYL as a technology probe [42] to achieve three objectives: (1) field-test ALgANYL to validate our material characterization and design space, (2) gain insight into the needs and wants of the users working with AlganyL, and (3) inspire creators to think of potential future uses and applications for ALGANYL.

\subsection{Technology Probes}

Technology probes are an interdisciplinary method for co-designing technologies with users, combining engineering, social science, and design, to understand how users interact with and ultimately utilize new technologies [42]. This method is rooted in participatory design, which encourages users to actively be a part of the design process [87]. It uses experience prototyping/bodystorming, which involves informal, kinesthetic interactions throughout the prototyping process $[18,64,86]$. We leveraged experience prototyping to 
achieve our first objective: field-testing AlgANYL. By having users make Alganyl in their own homes and explore applications of their choosing, we gained insight into the accessibility of ALGANYL as a material to make at home and began to verify our material characterization and design space. To achieve our second objective of understanding the user's attitudes towards designing with ALGANYL, we included a survey containing questions to identify likes, dislikes, and general feelings about designing with AlgAnYL (see survey in Supplementary Material). To achieve our third objective of inspiring users to come up with future uses for ALGANYL given their experiences making ALGANYL, we took inspiration from cultural probes; specifically, Shewbridge et al.'s cultural probe prompts that ask users to think about AlgANYL objects that can be given as gifts, and objects that can be repaired, customized, or modified with Alganyl [89]. This type of cultural probing is key to identifying real-world future applications for new technologies based on the user's experiences and lives [35, 37, 89].

\subsection{Participants}

We conducted a user study with twelve participants (seven female and five male, 18-30 years), all of whom were university students recruited through two university classes on design: a creative technologies course and a biodesign course. These courses consisted of experienced environmental design and product design students. All participants expressed interest in sustainability, biodesign, and future technologies, and thus they were familiar with the concept of bioplastics. However, only two of the participants had prior experience with making bioplastics. All participants voluntarily joined our study and were not pressured to participate by their instructor nor with grades.

\subsection{Materials}

Participants received an Alganyl kit containing: $10 \mathrm{~g}$ of agar powder, $15 \mathrm{~g}$ of glycerin, 1 circular piece of ready-made ALGANYL of 40 $\mathrm{cm}$ in diameter, and written online instructions for how to make Alganyl at home. The materials provided were sufficient for making a circular piece of AlgANYL approximately $40 \mathrm{~cm}$ in diameter, and more materials were available to the participants upon request.

\subsection{Procedure}

The nature of the participants' remote courses prompted us to utilize a safe learning environment that was university policy during the COVID-19 pandemic. We conducted an online workshop during which participants first listened to a 30-minute lecture about the cooking and handling process of ALGANYL, followed by a 30-minute hands-on experience with the material. By introducing the material to participants in their own homes, we also intended to achieve two points: (1) encourage participants to imagine the potentials of creating their own bioplastics while avoiding the association of it to a rigid university environment; and (2) observe behaviors and struggles of participants with a new material in a more familiar environment.

The lecture had three parts: first, the researcher introduced $\mathrm{AL}-$ GANYL as a biodegradable, plastic-like material and explained the ingredients and making process of ALGANYL described in Section
3.1. Next, the participants were shown Alganyl samples of various colors and textures seen in Section 4, and lastly shown our applications-the floral sculpture (Figure 5), the doll clothing (Figure 8), the UV indicator bracelet (Figure 10), the thermosensing necklace (Figure 11), and the recycled concert bag (Figure 13).

The lecture was then followed by a hands-on workshop, during which the participants individually made ALgANYL in their homes using either a microwave or stove-top. After the workshop, the participants were invited to explore the material and, if possible, design and make an application of their choosing. The participants were given the option to make their application from the pre-made Alganyl piece provided in their kit and/or from the Alganyl they made during the workshop. The participants were encouraged to use any additional ingredients (e.g., dyes, conductive materials, sensing pigments) and fabrication tools they had access to at home (e.g., scissors, fabric irons).

Last, the participants reported their experiences and further thoughts via a survey (see survey in Supplementary Material). The survey first asked participants to submit a photo and short description of their made AlganYL application. Then, the participants had to answer a total of six 6-point Likert scale questions about their experience designing and making with AlganyL. The scale ranged from strongly disagree to strongly agree. The second part of the survey had ten open-ended questions that prompted participants to reflect on their experiences making their ALGANYL applications and envision potential future applications for ALGANYL. The participants were encouraged to reference their everyday lives and experiences to speculate new possibilities for AlganyL. The whole process-from the introductory lecture to submitting the surveylasted an average of two weeks for the participants.

\subsection{Findings}

We obtained the data presented in this section from observed behavior and answers to the survey conducted post-workshop. We organize our findings based on our three objectives for our user study: (1) field test Alganyl, (2) gain insight into the user's needs and desires, and (3) inspire future applications for Alganyl. The researchers reviewed the participants' made and suggested applications, then used open coding analysis to discern primary object categories (using a broader version of Amazon's classification of household objects [5]). To further analyze the qualitative data regarding the participants' experiences, behavior, and attitudes, we organized our observation notes and open-ended responses based on our three objectives.

Field Test. During the workshop, we observed that the participants readily wanted to succeed in making ALganyl that resembled the finished pieces provided to them in their kits. While some participants looked confident in their kitchens and seemed to innately understand when the ALGANYL was thoroughly cooked and ready to be poured and dried, other participants asked many questions, holding up their mixtures of ALgANYL to their computer cameras to get reassurance. We think that these behaviors most likely come from a familiarity with working in the kitchen. Users who actively cook seemed to have more embodied knowledge about cooking Alganyl than users who do not spend much time in the kitchen. Eight out of the twelve participants felt that it was easy to make 
their intended AlganYL product. In comparison, ten participants out of the twelve indicated that their product turned out the way they intended. These observations tell us that ALGANYL was easy to work with overall and that participants generally had positive reactions to the experience. When asked what characteristics of Alganyl that was important to keep, a number of participants pointed out the ease of use: "Easy to make, doesn't require special equipment, you can play with changing the ratio of the ingredients and get different materials" $-P 8$

Participants' attitudes and responses after the workshop shows that they have a decent preliminary understanding of the capabilities of AlgAnyL, with some exceptions. By handling the material themselves, they were able to deduce its potential for fashioning flexible products, like grocery bags or cling film. However, some participants inaccurately suggested that ALGANYL would be a fitting choice for creating bottles or Tupperware containers; ALGANYL does not have the rigidity required for such objects. Thus, additional experimentation may be required to intuit its uses correctly.

We observed that the ALGANYL applications made by the participants fell into three categories: experimental AlgAnyL swatches that explored specific fabrication techniques (Figure 15a), utilitarian objects (Figure 15b), and specialty/creative items (Figure 15c). Three participants created new design methods (molding (Figure 15a), weaving, and patterning texture), four created utilitarian objects (detergent pods (Figure 15b), bowl covers, wine bottle covers, and bags). Five participants created specialty items (eye masks (Figure 15c), clothing, eucalyptus band-aids, instant food, and bracelets). Participants were able to deduce Alganyl's potential to implement sustainable practices. This ability is evident in the products created during the workshop. Objects such as bags, instant food packaging, and detergent pods suggest participants strive for sustainability. Moreover, we speculate that these practical applications, especially bags, would be easy to scale up to a commercial level for widespread use.

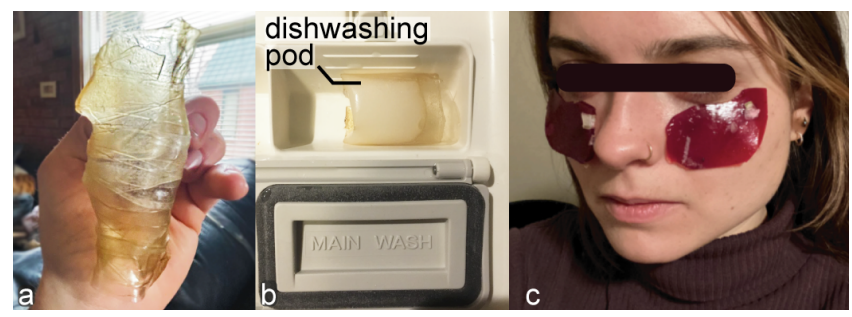

Figure 15: Participants made Alganyl applications that we categorized as experiments, utilitarian objects, and specialty items. Examples include (a) a heat molding experiment, (b) a dishwasher detergent pod, and (c) eye masks infused with hibiscus and liquid collagen. The photos were taken by the participants and used with permission.

User Feedback - Desirable Features. Participants' experiences with cooking and designing with ALGANYL allowed them to identify aspects of the material that they enjoyed. When asked what characteristics of ALganyl they would like to keep, participants favored its biodegradability (they learned about that during the lecture), flexibility and simplicity to make. Some participants specifically named this feature as the one they cared to maintain. Their interest in biodegradability is also evident in the overwhelming number of suggestions for replacing common single-use plastics with ALGANYL. Participants also highlighted flexibility and malleability as vital characteristics:

"Flexibility is crucial, most develop-able surfaces are like paper, and alganyl is very formable." -P1

"I love the the ability to be both flexible and durable/strong." -P11

Another reoccurring feature participants wanted to preserve is the simplicity of making ALGANYL. When asked what characteristics of Alganyl participants want to keep, Participant 2 responded, "I liked that it didn't require a lot of ingredient [s]." Participant 7 stated, "Ease of cooking and manipulating/heat fusing." It is important to note that when processes and systems are more accessible (i.e., easier to use, understand, and access), it is easier to encourage environmental consciousness [9,36].

User Feedback - Barriers of Use. Identifying potential deterrents to creating or using ALGANYL can help improve the usability of the material and provide insights into how to incorporate this or other bioplastics into everyday life. Most participants reported that they found Alganyl easy to work with, though when asked on a 6-point Likert scale whether they felt there was a material barrier to working with it, half of them confirmed that there was. These responses indicate that they might have felt limited by the material's abilities. While most participants did not feel there was a knowledge barrier in working with ALgANyL, four out of twelve felt there was. Participant 11 noted specifically that they would like to "have a clearer knowing of the agar/glycerin ratio to get specific desired results." This response indicates that instruction should more clearly emphasize the customizability of the material based on ingredient ratio.

Five participants also noted AlganyL's inability to resist water for long periods of time as a characteristic they would like to change. This suggests that people would be less inclined to use the material for any purposes that may involve the product getting wet. Although this was a barrier for some participants, we were surprised to see other participants leverage this property by creating dishwasher pods and face masks that dissolve in water.

Future Applications. When filling the survey, the participants suggested potential future applications for ALGAnYL. We asked participants four questions about potential applications they could make with ALGANYL: (1) List 5 gifts that you could give a friend/loved one made out of ALGANYL; (2) List 5 items in your home that you could customize or modify with Alganyl; (3) List 5 items broken items in your home that could be repaired or replaced with ALGANYL; (4) Are there any other applications that you could make from ALGANYL?

From these questions, we received 183 potential applications (including repeats), 118 of which were unique. Table 2 showcases the number of applications in each object category for each prompt. Our object categories include Clothes \& Accessories (e.g., clothes, jewelry, purses, wallets, phone cases, etc.), Art \& Decor (e.g., wall art, furniture, curtains, lampshades, sculptures, etc.), Utilitarian Objects (e.g., cups, bowls, Ziploc bags, packaging, saran wrap, etc.), and Other (e.g., face masks, guitar picks, gardening paraphernalia, edible bioplastics, medical applications, etc.). The most notable observation from these results is the number of utilitarian objects 


\begin{tabular}{ccccc}
\hline Prompt & Clothes \& Accessories & Art \& Decor & Utilitarian Objects & Other \\
\hline Give/Gift & 22 & 13 & 12 & 6 \\
Customize/Modify & 9 & 11 & 17 & 5 \\
Repair/Replace & 7 & 5 & 39 & 5 \\
Other & 3 & 7 & 7 & 15
\end{tabular}

Table 2: Number of applications thought of for each prompt categorized by type of object.

made from single-use plastics that participants wanted to replace with Alganyl. These suggested applications again emphasize the desire to incorporate sustainability in daily life, as these applications support the replacement of harmful or single-use plastics.

\section{DISCUSSION}

\subsection{Limitations of AlganyL}

While we compare Alganyl to vinyl fabric in Section 3.3, we must note that ALGANYL is not as durable and therefore cannot be used as often or as long as vinyl. However, ALgANYL can be re-cooked at any time to give new life to the material. As for the biodegradability of ALGANYL, the degradation rate depends on the environment of the soil it is placed in (temperature, humidity, microbes, etc.) and the piece of AlganYL itself. While we make an effort to embed biodegradable materials (e.g., activated charcoal, natural dye, etc.), we acknowledge that many designers might want to embed non-biodegradable elements such as LEDs, in which case we are strong proponents of re-use. Similarly, ALGANYL faces limitations in external environments-colored ALGAnYL fades when placed in the sun, and cold water like rain and snow deform ALGANyL when redried (waterproofing being a property many participants desired). In these scenarios, we highly recommend taking advantage of the re-cooking process to re-make the ALGANYL applications that have been negatively impacted.

\subsection{Experiences with Alganyl}

This work highlights the importance of experimentation and experience prototyping [18] during the design and making process, which we employed in order to characterize the material (Section 3) and explore the design space (Section 4). Most of our initial experiments, which centered around optimizing the ratio of ingredients, relied on kinesthetic-based prototyping as described by Loke [64]. By focusing on the feel of each ALganyl sample and observing how AlgANYL reacted to our interactions and movements, we gained a deep tacit knowledge about the material. Our 'gut' reactions regarding each sample of ALGANYL paired with our tensile testing result led us to our optimal recipe. Once we optimized AlganyL, we experimented more with creating samples that looked aesthetically pleasing. We built upon our experiments described in the design space, using our reactions from previous experiences with Alganyl and intuition to guide us towards new possibilities. We also noticed that the implicit knowledge about the material gained through our physical interactions provided us with an almost intuitive understanding of what kind of applications ALganyL could be used for (i.e., bodystorming [86]). Meanwhile, the participants in our study did not nearly spend as much time physically interacting with the material-our experiences spanning over months while theirs spanning over only two weeks. However, most participants quickly understood the limitations and possibilities by cooking their own Alganyl and attempting to make their own Alganyl applications.

In the context of personal, at-home fabrication and DIY, the sensory and aesthetic qualities of ALGANYL depend on each maker. However, unlike more common at-home fabrication methods like 3D printing, making AlganYL is a much more involved and embodied process. It is adjacent to slow design [38]-the maker has to wait for cooking and drying times while also considering design decisions like color, texture, flexibility, and durability at each step. This slower process requires the maker to be even more engaged, affording real-time sensory feedback when cooking and fabricating Alganyl. This experience opens up new considerations regarding the customizability of Alganyl. For example, our user study asked participants to make custom AlgANYL applications. It is important to note that not only were their applications custom, but the ALGANYL itself they used was custom-made by them, reflecting the participants' experience (or lack of experience) and process of creating the material. Unlike cultural probes that traditionally provide a "complete" artifact and task to get a glimpse into the participants' lives [35], we essentially examine two artifacts: the AlgANYL and the Alganyl application. Essentially, the two steps of cooking ALGANYL and then fabricating an application encouraged participants to engage with AlganYL on a deeper level, asking them to invest time, effort, thought, and resources.

\subsection{Implications of AlganyL}

AlgANYL provides an accessible entry point into the world of biomaterials. We cook Alganyl using equipment (i.e., stovetop or microwave) found in most kitchens and ingredients easily found at grocery stores at relatively low cost. This makes ALgANYL incredibly accessible from a materials standpoint (ALgANYL costs approximately USD 0.03 per kilogram). Moreover, the intuitive act of cooking and the familiarity of the kitchen as a creative environment makes Alganyl an un-intimidating material to create. Participants in our study particularly liked the simplicity and ease of making Alganyl at home. As such, Alganyl would be a great introduction to biomaterials for designers that are hesitant to work with such materials because of their lack of biology knowledge and training.

In addition, Alganyl could be used to raise designers' awareness about sustainability in their practices and direct them towards biodegradable and/or easily recyclable biomaterials. Thus, we encourage shifting away from the traditional human-centered design goals [72] that dominate HCI towards more-than-human design goals that consider the interdependencies of humans and other living things $[24,90]$. We see Alganyl particularly impacting the 
ways designers and HCI researchers prototype, thinking of the "life cycle" of materials when creating prototypes that could be easily re-cooked and re-fabricated into another prototype, encouraging lots of iterations without impacting the environment. Moreover, by leveraging the comfortable environment of the kitchen and the familiarity of cooking, Alganyl could engage people beyond the design and HCI communities with biomaterials and sustainable fabrication. By involving a larger community, we can begin to speculate on a future where not just expert designers, but everyone can fabricate single-use plastic items such as sandwich bags and saran wrap in their own homes-ultimately minimizing petroleum-based plastic waste at large.

\section{CONCLUSION}

This work explores designing with ALgANYL: a vinyl-like biomaterial that can be customized in a variety of ways to make unique and sustainable applications. Specifically, we present a design space for ALGANYL using a recipe optimized for strength and flexibility. We showcase our explorations through a set of applications, such as a sculpture, doll clothing, environment-sensing accessories, and a recycled concert bag. Last, we conducted a user study with 12 participants that field tests Alganyl as an accessible biomaterial, examines the desirable features and drawbacks of AlgANyL, and identifies potential future applications for Alganyl. The participants created applications ranging from strictly functional (e.g. detergent pods and bowl covers) to highly artistic (e.g., eye masks and bracelets). The participants appreciated the accessibility of the design process (i.e., the ease of making and using AlgANYL) and the biodegradability of the material. Ultimately, we noticed a strong interest from the participants to have access to more sustainable alternatives to current plastics and a willingness to make their biomaterials at home. As future work, we plan to explore applications that leverage some of the unique properties of our material, e.g., take advantage of ALGANYL's ability to melt in boiling water to create edible noodles packaging. Another example is leveraging the fact that ALGANYL shrinks (while drying after having been recooked or after prolonged water exposure) to create shape-changing interfaces. Lastly, we plan to run studies that include experienced biodesigners.

\section{ACKNOWLEDGMENTS}

We would like to acknowledge Netta Ofer for helping with photos and feedback.

\section{REFERENCES}

[1] 2020. Blokus. Retrieved December 12, 2020 from https://www.mattelgames. com/en-us/strategy/blokus

[2] Jekaterina Aleksejeva, Paul Biedermann, Iulia Gavriliuc, Ona Orlovaite, and Danielle Wilde. 2019. Crafting bioplastics: Materially reconfiguring everyday food practices. In Proceedings of the 4th Biennial Research Through Design Conference 19-22/03/2019. RTD Conference.

[3] AlgiKnit. 2020. Retrieved November 3, 2020 from https://www.algiknit.com

[4] Mirela Alistar and Margherita Pevere. 2020. Semina Aeternitatis: Using Bacteria for Tangible Interaction with Data. In Extended Abstracts of the $2020 \mathrm{CHI}$ Conference on Human Factors in Computing Systems. 1-13.

[5] Amazon. 2021. Overview of Categories. Retrieved June 10, 2021 from https: //sellercentral.amazon.com/gp/help/external/G200332540?language=en_US

[6] ASTM. 2014. Standard Test Method for Tensile Properties of Plastics.

[7] Christoph Bader, William G Patrick, Dominik Kolb, Stephanie G Hays, Steven Keating, Sunanda Sharma, Daniel Dikovsky, Boris Belocon, James C Weaver,
Pamela A Silver, et al. 2016. Grown, printed, and biologically augmented: An additively manufactured microfluidic wearable, functionally templated for synthetic microbes. 3D Printing and Additive Manufacturing 3, 2 (2016), 79-89.

[8] Jordan Baker. 2018. Kombucha jacket lands HSC student Heather in the London College of Fashion. The Sydney Morning Harold (2018).

[9] Margaret Banga. 2011. Household knowledge, attitudes and practices in solid waste segregation and recycling: the case of urban Kampala. Zambia Social Science Journal 2, 1 (2011), 4

[10] Valery A Barachevsky. 2020. 2D materials for smart energochromic sunscreen devices. In 2D Nanomaterials for Energy Applications. Elsevier, 173-208.

[11] Bahareh Barati, Elvin Karana, Sylvia Pont, and Tim van Dortmont. 2021. LIVING LIGHT INTERFACES-AN EXPLORATION OF BIOLUMINESCENCE AESTHETICS. In Designing Interactive Systems Conference 2021. 1215-1229.

[12] Axel. Barrett. July 8, 2019. First Clothes Made From Bioplastics. Retrieved November 3, 2020 from https://bioplasticsnews.com/2019/07/08/first-clothesmade-from-bioplastics/

[13] Fiona Bell, Alice Hong, Andreea Danielescu, Aditi Maheshwari, Ben Greenspan, Hiroshi Ishii, Laura Devendorf, and Mirela Alistar. 2021. Self-deStaining Textiles: Designing Interactive Systems with Fabric, Stains and Light. In Proceedings of the 2021 CHI Conference on Human Factors in Computing Systems. 1-12.

[14] Michael Bernstein and Michael Woods. 2009. Plastics in oceans decompose, release hazardous chemicals, surprising new study says. Retrieved November 21 (2009), 2016.

[15] Joanna Berzowska and Marcelo Coelho. 2006. Smoks: the memory suits. In CHI'06 Extended Abstracts on Human Factors in Computing Systems. 538-543.

[16] Eli Blevis. 2007. Sustainable interaction design: invention \& disposal, renewal $\&$ reuse. In Proceedings of the SIGCHI conference on Human factors in computing systems. 503-512.

[17] BoltThreads. 2021. Mylo. Retrieved April 12, 2021 from https://boltthreads. com/technology/mylo/

[18] Marion Buchenau and Jane Fulton Suri. 2000. Experience prototyping. In Proceedings of the 3 rd conference on Designing interactive systems: processes, practices, methods, and techniques. 424-433.

[19] Jesse L Butterfield, Sean P Keyser, Karan V Dikshit, Hyejin Kwon, Maranke I Koster, and Carson J Bruns. 2020. Solar Freckles: Long-Term Photochromic Tattoos for Intradermal Ultraviolet Radiometry. ACS nano 14, 10 (2020), 1361913628.

[20] Adrian David Cheok, Roger Thomas Kok, Chuen Tan, Owen Noel Newton Fernando, Tim Merritt, and Janyn Yen Ping Sen. 2008. Empathetic living media. In Proceedings of the 7th ACM conference on Designing interactive systems. 465-473.

[21] Jasmin Malik Chua. 2015. Grow Your Own Microbial "Leather" in Your Kitchen (DIY Tutorial). Retrieved March 2, 2021 from http://www.ecouterre.com/growyour-ownmicrobial-leather-in-your-kitchen-diy-tutorial/

[22] Nate J Cira, Alice M Chung, Aleksandra K Denisin, Stefano Rensi, Gabriel N Sanchez, Stephen R Quake, and Ingmar H Riedel-Kruse. 2015. A biotic game design project for integrated life science and engineering education. PLoS Biol 13, 3 (2015), e1002110.

[23] Evo \& Co. 2021. Evoware. Retrieved April 12, 2021 from https://rethinkplastic.com/brand/evoware/

[24] Paul Coulton and Joseph Galen Lindley. 2019. More-than human centred design: Considering other things. The Design fournal 22, 4 (2019), 463-481.

[25] Laura Devendorf, Joanne Lo, Noura Howell, Jung Lin Lee, Nan-Wei Gong, M Emre Karagozler, Shiho Fukuhara, Ivan Poupyrev, Eric Paulos, and Kimiko Ryokai. 2016. "I don't Want to Wear a Screen" Probing Perceptions of and Possibilities for Dynamic Displays on Clothing. In Proceedings of the 2016 CHI Conference on Human Factors in Computing Systems. 6028-6039.

[26] Dezeen. November 5, 2019. Charlotte McCurdy creates "carbon-negative" raincoat from algae bioplastic. Retrieved November 3, 2020 from https: //www.dezeen.com/2019/11/05/charlotte-mccurdy-bioplastic-raincoat-2/

[27] DIYBio. 2020. An Institution for the Do-It-Yourself Biologist. Retrieved September 16, 2020 from https://diybio.org

[28] Sandra Domenek, Pierre Feuilloley, Jean Gratraud, Marie-Hélène Morel, and Stéphane Guilbert. 2004. Biodegradability of wheat gluten based bioplastics. Chemosphere 54, 4 (2004), 551-559.

[29] Jeffrey S Dugan. 2001. Novel properties of PLA fibers. International Nonwovens Journal 3 (2001), 1558925001OS-01000308.

[30] Margaret Dunne. 2018. Bioplastic Cook Book. Retrieved December 12, 2020 from https://issuu.com/nat_arc/docs/bioplastic_cook_book_3

[31] Ecovative. 2021. MycoFlex. Retrieved April 12, 2021 from https:// ecovativedesign.com/mycoflex

[32] Karen El Asmar. 2019. Social Microbial Prosthesis: Towards Super-Organism Centered Design. In Extended Abstracts of the 2019 CHI Conference on Human Factors in Computing Systems. 1-7.

[33] S Mehdi Emadian, Turgut T Onay, and Burak Demirel. 2017. Biodegradation of bioplastics in natural environments. Waste management 59 (2017), 526-536.

[34] Cherise Fong. 2017. Paint your own circuits with DIY conductive ink. Retrieved April 12, 2021 from https://www.makery.info/en/2017/02/06/peignezvos-circuits-a-lencre-conductrice-diy/ 
[35] Bill Gaver, Tony Dunne, and Elena Pacenti. 1999. Design: cultural probes. interactions 6, 1 (1999), 21-29.

[36] Andrea Gossett, Andrea Gossett, Mansha Mirza, Ann Kathleen Barnds, and Daisy Feidt. 2009. Beyond access: A case study on the intersection between accessibility, sustainability, and universal design. Disability and Rehabilitation: Assistive Technology 4, 6 (2009), 439-450.

[37] Connor Graham and Mark Rouncefield. 2008. Probes and participation. In Proceedings of the Tenth Anniversary Conference on Participatory Design 2008. 194-197.

[38] Barbara Grosse-Hering, Jon Mason, Dzmitry Aliakseyeu, Conny Bakker, and Pieter Desmet. 2013. Slow design for meaningful interactions. In Proceedings of the SIGCHI Conference on Human Factors in Computing Systems. 3431-3440.

[39] Siew-Ling Hii, J Lim, Wan-Teck Ong, and Ching-Lee Wong. 2016. Agar from Malaysian red seaweed as potential material for synthesis of bioplastic film. 7 Eng. Sci. Technol 11 (2016), 1-15.

[40] David Holstius, John Kembel, Amy Hurst, Peng-Hui Wan, and Jodi Forlizzi. 2004 Infotropism: living and robotic plants as interactive displays. In Proceedings of the 5th conference on Designing interactive systems: processes, practices, methods, and techniques. 215-221.

[41] Cedric Honnet, Hannah Perner-Wilson, Marc Teyssier, Bruno Fruchard, Jürgen Steimle, Ana C Baptista, and Paul Strohmeier. 2020. PolySense: Augmenting Textiles with Electrical Functionality using In-Situ Polymerization. In Proceedings of the 2020 CHI Conference on Human Factors in Computing Systems. 1-13.

[42] Hilary Hutchinson, Wendy Mackay, Bo Westerlund, Benjamin B Bederson, Allison Druin, Catherine Plaisant, Michel Beaudouin-Lafon, Stéphane Conversy, Helen Evans, Heiko Hansen, et al. 2003. Technology probes: inspiring design for and with families. In Proceedings of the SIGCHI conference on Human factors in computing systems. 17-24.

[43] Chromatic Technologies Inc. 2021. Color Changing Inks. Retrieved April 12 2021 from https://www.ctiinks.com/

[44] Instructables. 2021. Retrieved June 16, 2021 from https://www.instructables com/

[45] Yuhua Jin, Isabel Qamar, Michael Wessely, and Stefanie Mueller. 2020. Photochromeleon: Re-programmable multi-color textures using photochromic dyes. In ACM SIGGRAPH 2020 Emerging Technologies. 1-2.

[46] Joann. 2021. 8 Gauge Vinyl 54" Clear. Retrieved May 15, 2021 from https: //www.joann.com/8-gauge-vinyl-54in-clear/1068691.html

[47] Viirj Kan, Katsuya Fujii, Judith Amores, Chang Long Zhu Jin, Pattie Maes, and Hiroshi Ishii. 2015. Social textiles: Social affordances and icebreaking interactions through wearable social messaging. In Proceedings of the Ninth International Conference on Tangible, Embedded, and Embodied Interaction. 619624.

[48] Elvin Karana, Bahareh Barati, Valentina Rognoli, Anouk Zeeuw Van Der Laan, et al. 2015. Material driven design (MDD): A method to design for material experiences. (2015).

[49] Honesty Kim, Lukas Cyrill Gerber, Daniel Chiu, Seung Ah Lee, Nate J Cira Sherwin Yuyang Xia, and Ingmar H Riedel-Kruse. 2016. LudusScope: accessible interactive smartphone microscopy for life-science education. PloS one 11, 10 (2016), e0162602.

[50] Raphael Kim, Siobhan Thomas, Roland van Dierendonck, and Stefan Poslad. 2018. A new mould rush: designing for a slow bio-digital game driven by living micro-organisms. In Proceedings of the 13th International Conference on the Foundations of Digital Games. 1-9.

[51] David Sun Kong, Keerthi Shetty, Thrasyvoulos Karydis, Yixiao Jiang, Alexandria Guo, Matt Mendoza, Mary Tsang, Shannon Johnson, Ani Liu, Viirj Kan, and Rachel Smith. 2016. Biota Beats. Retrieved May 15, 2021 from www.biotabeats. org

[52] Manuel Kretzer. [n.d.]. Bioplastics. Retrieved April 12, 2021 from http:// materiability.com/portfolio/bioplastics-2/

[53] Satoshi Kuribayashi and Akira Wakita. 2006. PlantDisplay: turning houseplants into ambient display. In Proceedings of the 2006 ACM SIGCHI international conference on Advances in computer entertainment technology. 40-es.

[54] Stacey Kuznetsov, Cassandra Barrett, Piyum Fernando, and Kat Fowler. 2018. Antibiotic-responsive bioart: Exploring DIYbio as a design studio practice. In Proceedings of the 2018 CHI Conference on Human Factors in Computing Systems. $1-14$.

[55] Stacey Kuznetsov, Carrie Doonan, Nathan Wilson, Swarna Mohan, Scott E Hudson, and Eric Paulos. 2015. DIYbio things: open source biology tools as platforms for hybrid knowledge production and scientific participation. In Proceedings of the 33rd Annual ACM Conference on Human Factors in Computing Systems. 4065-4068.

[56] Stacey Kuznetsov and Eric Paulos. 2010. Rise of the expert amateur: DIY projects, communities, and cultures. In Proceedings of the 6th Nordic conference on human computer interaction: extending boundaries. 295-304.

[57] Oi-Ying Kwong. 2011. Bio-Plastic Handbook. Retrieved April 12, 2021 from https://issuu.com/oi-ying/docs/bio-plastic handbook2

[58] Pirjo Kääriäinen, Liisa Tervinen, Tapani Vuorinen, and Nina Riutta. 2020. The Chemarts Cookbook. Retrieved April 12, 2021 from https://digitalcommons. risd.edu/library e-resources/14

[59] Counter Culture Labs. 2021. A Community Supported Microbiology Maker Space. Retrieved March 2, 2021 from https://www.counterculturelabs.org/

[60] Seung Ah Lee, Engin Bumbacher, Alice M Chung, Nate Cira, Byron Walker, Ji Young Park, Barry Starr, Paulo Blikstein, and Ingmar H Riedel-Kruse. 2015. Trap it! A playful human-biology interaction for a museum installation. In Proceedings of the 33rd Annual ACM Conference on Human Factors in Computing Systems. 2593-2602.

[61] YA Lee. 2016. Case study of renewable bacteria cellulose fiber and biopolymer composites in sustainable design practices. In Sustainable Fibres for Fashion Industry. Springer, 141-162.

[62] Notpla Limited. 2021. Ooho! Retrieved April 12, 2021 from https://www.notpla. com/products- $2 /$

[63] Open Myco LLC. 2021. GROW.bio. Retrieved April 12, 2021 from https: //grow.bio/

[64] Lian Loke and Toni Robertson. 2013. Moving and making strange: An embodied approach to movement-based interaction design. ACM Transactions on Computer-Human Interaction (TOCHI) 20, 1 (2013), 1-25.

[65] Laura Luchtman and Ilfa Siebenhaar. 2018. Living Color. Retrieved May 15, 2021 from https://livingcolour.eu

[66] M Nizar Machmud, Reza Fahmi, Rohana Abdullah, and Coco Kokarkin. 2013. Characteristics of red algae bioplastics/latex blends under tension. International fournal of Science and Engineering 5, 2 (2013), 81-88.

[67] Jennifer C Mankoff, Eli Blevis, Alan Borning, Batya Friedman, Susan R Fussell, Jay Hasbrouck, Allison Woodruff, and Phoebe Sengers. 2007. Environmental sustainability and interaction. In CHI'07 extended abstracts on Human factors in computing systems. 2121-2124.

[68] Materiom. 2021. Materials Library. Retrieved May 15, 2021 from https:// materiom.org/

[69] Kristen McLaughlin, Allison Webb, Kaitlin Bratt, and Daniel Saloni. 2020. Bioplastic Modified with Woodflour for Additive Manufacturing. In International Conference on Applied Human Factors and Ergonomics. Springer, 86-94.

[70] William Myers. 2012. Bio design. Museum of Modern Art.

[71] Audrey Ng. 2017. Grown microbial 3D fiber art, Ava: fusion of traditional art with technology. In Proceedings of the 2017 ACM International Symposium on Wearable Computers. 209-214.

[72] Don Norman. 2013. The design of everyday things: Revised and expanded edition. Basic books.

[73] Netta Ofer, Fiona Bell, and Mirela Alistar. 2021. Designing Direct Interactions with Bioluminescent Algae. In Designing Interactive Systems Conference 2021 (DIS '21).

[74] Maggie Orth, Rehmi Post, and Emily Cooper. 1998. Fabric computing interfaces. In CHI 98 conference summary on Human factors in computing systems. 331-332.

[75] Neri Oxman. 2020. Material Ecology. Retrieved December 12, 2020 from https://www.moma.org/calendar/exhibitions/5090

[76] Stefano Parisi, Valentina Rognoli, and Marieke Sonneveld. 2017. Material Tinkering. An inspirational approach for experiential learning and envisioning in product design education. The Design fournal 20, sup1 (2017), S1167-S1184.

[77] Pat Pataranutaporn, Jaime Sanchez De La Vega, Abhik Chowdhury, Audrey Ng, and Galina Mihaleva. 2018. Toward Growable Robot: Exploring and Integrating Flexible-Biological Matter with Electronics. In 2018 International Flexible Electronics Technology Conference (IFETC). IEEE, 1-4.

[78] Owain Pedgley, Valentina Rognoli, and Elvin Karana. 2016. Materials experience as a foundation for materials and design education. International fournal of Technology and Design Education 26, 4 (2016), 613-630.

[79] Roshan Lalintha Peiris, Owen Noel Newton Fernando, Chua Su Bee, Adrian David Cheok, Arij Glycin Ganesan, and Prabhash Kumarasinghe. 2011. dMarkers: ubiquitous dynamic makers for augmented reality. In Proceedings of the 10th International Conference on Virtual Reality Continuum and Its Applications in Industry. 217-224.

[80] Juliette Pepin. 2014. Research Book Bioplastics. Retrieved April 12, 2021 from https://issuu.com/juliettepepin/docs/bookletbioplastic

[81] Cecilia Raspanti. 2020. BioFabricating Materials. Retrieved April 12, 2021 from https://class.textile-academy.org/classes/2020-21/week06/

[82] Christian Remy, Oliver Bates, Vanessa Thomas, and Elaine M Huang. 2017. The limits of evaluating sustainability. In Proceedings of the 2017 Workshop on Computing Within Limits. 103-110.

[83] Hannah Ritchie and Max Roser. 2018. Plastic pollution. Our World in Data (2018).

[84] Erica Robles and Mikael Wiberg. 2010. Texturing the" material turn" in interaction design. In Proceedings of the fourth international conference on Tangible, embedded, and embodied interaction. 137-144.

[85] Renata Ferreira Santana, Renata Cristina Ferreira Bonomo, Olga Reinert Ramos Gandolfi, Luciano Brito Rodrigues, Leandro Soares Santos, Ana Clarissa dos Santos Pires, Cristiane Patrícia de Oliveira, Rafael da Costa Ilhéu Fontan, and Cristiane Martins Veloso. 2018. Characterization of starch-based bioplastics from jackfruit seed plasticized with glycerol. fournal of food science and technology 55, 1 (2018), 278-286. 
[86] Dennis Schleicher, Peter Jones, and Oksana Kachur. 2010. Bodystorming as embodied designing. Interactions 17, 6 (2010), 47-51.

[87] Douglas Schuler and Aki Namioka. 1993. Participatory design: Principles and practices. CRC Press.

[88] Alina S Sharova, Filippo Melloni, Guglielmo Lanzani, Christopher J Bettinger, and Mario Caironi. 2021. Edible electronics: The vision and the challenge. Advanced Materials Technologies 6, 2 (2021), 2000757.

[89] Rita Shewbridge, Amy Hurst, and Shaun K Kane. 2014. Everyday making: identifying future uses for 3D printing in the home. In Proceedings of the 2014 conference on Designing interactive systems. 815-824.

[90] Nancy Smith, Shaowen Bardzell, and Jeffrey Bardzell. 2017. Designing for cohabitation: Naturecultures, hybrids, and decentering the human in design. In Proceedings of the 2017 CHI Conference on Human Factors in Computing Systems. 1714-1725.

[91] Ana MM Sousa, Alberto M Sereno, Loic Hilliou, and Maria P Gonçalves. 2010 Biodegradable agar extracted from Gracilaria vermiculophylla: film properties and application to edible coating. In Materials science forum, Vol. 636. Trans Tech Publ, 739-744.

[92] Tom Spendlove. 2018. Are Makers Ready for DNA Bio-Display? Retrieved April 9, 2021 from https://www.engineering.com/story/are-makers-ready-fordna-bio-display

[93] Asma Tabassum. 2016. Biofilms from agar obtained from an agarophyte of Karachi coast. Pakistan fournal of Marine Sciences 25, 1\&2 (2016), 37-40.

[94] BROD \& TAYLOR. 2021. How to Grow a Kombucha Scoby - in just 10-12 Days. Retrieved April 9, 2021 from https://brodandtaylor.com/blogs/recipes/ kombucha-scoby/

[95] Eliangela de M Teixeira, Antônio AS Curvelo, Ana C Corrêa, José M Marconcini, Gregory M Glenn, and Luiz HC Mattoso. 2012. Properties of thermoplastic starch from cassava bagasse and cassava starch and their blends with poly (lactic acid). Industrial Crops and Products 37, 1 (2012), 61-68.

[96] Uyen Tran. 2021. Tomtex. Retrieved April 12, 2021 from https://www.tomtex.co/

[97] Joroen JG Van Soest, SHD Hulleman, D De Wit, and JFG Vliegenthart. 1996. Changes in the mechanical properties of thermoplastic potato starch in relation with changes in B-type crystallinity. Carbohydrate Polymers 29, 3 (1996), 225232.

[98] Eldy S Lazaro Vasquez and Katia Vega. 2019. From plastic to biomaterials: prototyping DIY electronics with mycelium. In Adjunct Proceedings of the 2019 ACM International foint Conference on Pervasive and Ubiquitous Computing and Proceedings of the 2019 ACM International Symposium on Wearable Computers. 308-311.

[99] Eldy S Lazaro Vasquez and Katia Vega. 2019. Myco-accessories: sustainable wearables with biodegradable materials. In Proceedings of the 23rd International Symposium on Wearable Computers. 306-311.

[100] Eldy S Lazaro Vasquez, Hao-Chuan Wang, and Katia Vega. 2020. Introducing the sustainable prototyping life cycle for digital fabrication to designers. In Proceedings of the 2020 ACM Designing Interactive Systems Conference. 13011312.

[101] Angela Vujic, Christopher Krause, Georgette Tso, Jiaqi Lin, Bicheng Han, and Pattie Maes. 2019. Gut-Brain Computer Interfacing (GBCI): Wearable Monitoring of Gastric Myoelectric Activity. In 2019 41st Annual International Conference of the IEEE Engineering in Medicine and Biology Society (EMBC). IEEE, 5886-5889.

[102] Lining Yao, Jifei Ou, Chin-Yi Cheng, Helene Steiner, Wen Wang, Guanyun Wang, and Hiroshi Ishii. 2015. bioLogic: Natto cells as nanoactuators for shape changing interfaces. In Proceedings of the 33rd Annual ACM Conference on Human Factors in Computing Systems. 1-10. 